\title{
Location, location, location: B-cell differentiation in the gut lamina propria
}

\author{
A Cerutti ${ }^{1,2}$
}

\section{The intestinal immune system includes an immunoglobulin (Ig)A-inductive site represented by Peyer's patches (PPs) and an IgA effector site represented by the lamina propria (LP). This canonical map of intestinal IgA production has been blurred recently by studies showing the presence of active IgA class switching in the LP. Here we discuss the functional implications and controversial nature of these findings.}

Immunoglobulin (Ig)A constitutes the most abundant antibody in the gut, where it provides immune protection through both high- and low-affinity binding systems (reviewed in ref. 1). While high-affinity IgA neutralizes toxins and pathogens, low-affinity IgA blocks the adhesion of commensal bacteria to epithelial cells. Remarkably, epithelial cells are required for IgA to fully perform its functions. Indeed, epithelial cells express a polymeric Ig receptor that shuttles IgA oligomers secreted by B cells from the LP onto the mucosal surface. Recent findings indicate that epithelial cells function not only as IgA transporters, but also as IgA inducers.

Unlike mouse B cells, which express only one IgA class, human B cells express two IgA subclasses. The large intestine contains predominantly IgA2, which is more resistant to digestion by bacteria than IgA 1 . We found that human colonocytes release the cytokine a proliferation-inducing ligand (APRIL), after sensing bacteria through Toll-like receptors. ${ }^{2}$ APRIL triggers direct
IgM-to-IgA2 class switch DNA recombination (CSR) and sequential IgA1-to-IgA2 CSR in LP B cells through a T-cell-independent (TI) mechanism that requires transcriptional activation of the $\mathrm{C} \alpha 2$ gene and subsequent expression of activationinduced cytidine deaminase (AID), a key component of the CSR machinery. Our data strongly suggest that epithelial cells utilize APRIL to enhance local production of protease-resistant IgA2, which is more suited than IgA1 to cope with the dense microbial community of the large intestine (Figure 1).

Similar to our work, elegant studies by Casellas and colleagues identified active IgA CSR in LP B cells from an AIDgreen fluorescent protein (AID-GFP) mouse indicator strain that recapitulates endogenous AID expression during the immune response. ${ }^{3}$ In this model, LP $B$ cells retain AID-GFP expression in the absence of mucosal germinal centers (GCs), a lymphoid microenvironment required for T-cell-dependent (TD) IgA responses. These data are consistent with earlier findings by Honjo and co-workers, ${ }^{4}$ who first documented TI IgA CSR in the mouse LP. In spite of this evidence, the contribution of the LP to intestinal IgA production remains controversial. ${ }^{5-7}$

Prior studies have generated a consensus over the existence of an IgA-inductive site represented by the organized lymphoid tissue of PPs and an IgA effector site represented by the non-organized lymphoid cells of the LP (reviewed in ref. 1). PPs typically give rise to monoreactive IgA antibodies with high affinity for TD antigens, such as complex proteins. This TD IgA response occurs in the GC of PPs and requires engagement of CD40 on conventional $\operatorname{IgM}^{+} \mathrm{B}$ cells (known as B-2 in mice) by CD40 ligand (CD40L) on $\mathrm{CD}^{+}{ }^{+} \mathrm{T}$-helper cells. Signals from CD40 lead to upregulation of AID, a DNA-editing enzyme essential for Ig gene diversification by CSR and somatic hypermutation. ${ }^{8}$ In general, CSR diversifies the antibody effector functions by substituting the heavy-chain constant region of IgM and IgD with that of IgG, IgA, or IgE, whereas somatic hypermutation introduces point mutations in the variable genes encoding the antigenbinding portion of Igs, thereby providing the structural correlate for selection by antigen of higher affinity mutants.

In PPs, CSR is predominantly skewed toward $\operatorname{Ig} \mathrm{A}$, possibly as a result of the intrinsic influence of their microenvironment (reviewed in ref. 1). Indeed, PPs contain abundant cytokines involved in IgA CSR and secretion, including interleukin (IL)-6, IL-10, and transforming growth factor- $\beta .{ }^{9}$ By upregulating guthoming receptors and under the influence of epithelial chemotactic factors, $\mathrm{IgA}^{+} \mathrm{B}$ cells migrate from PPs to the LP and thereafter differentiate into plasmablasts, which secrete large amounts of

${ }^{1}$ Department of Pathology and Laboratory Medicine of Weill Medical College of Cornell University, New York, New York, USA. ${ }^{2}$ Weill Graduate School of Medical Sciences of Cornell University, New York, New York, USA. Correspondence: A Cerutti (acerutti@med.cornell.edu) 


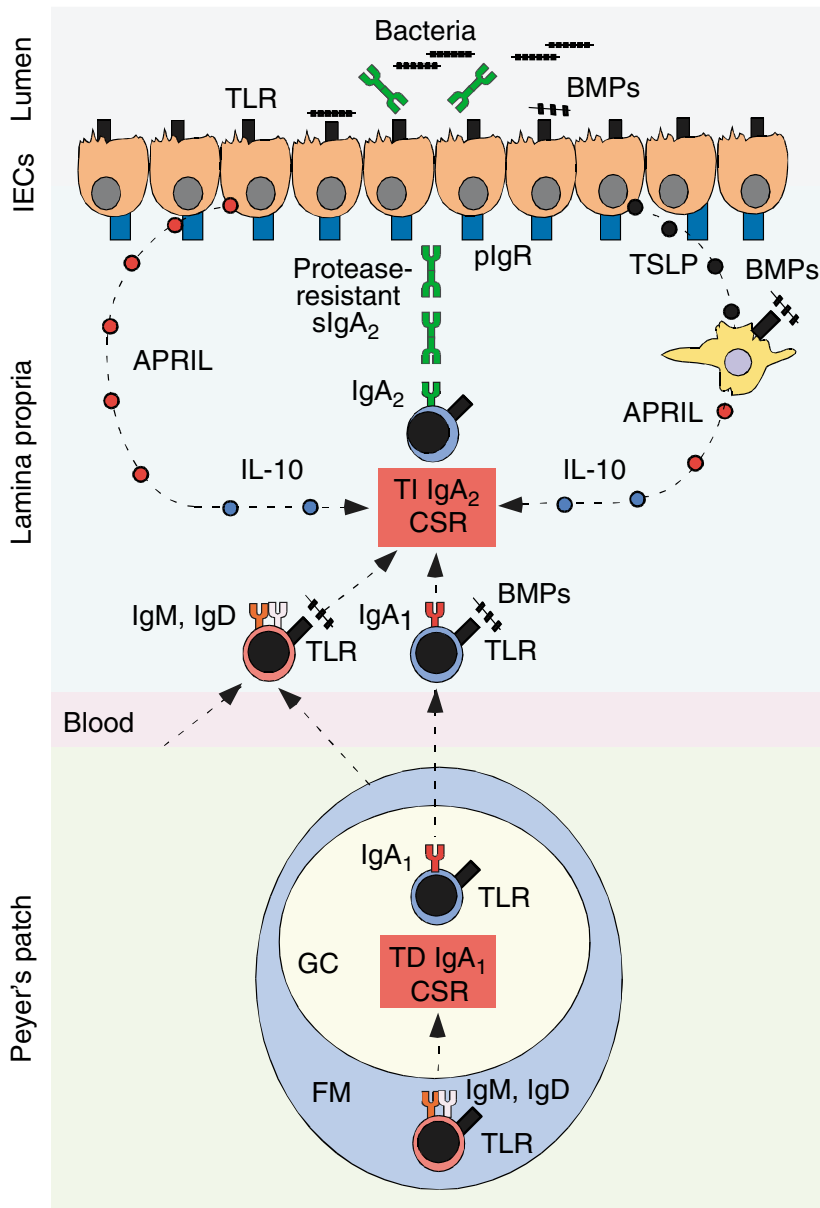

Figure 1 Proposed model of B-cell differentiation and IgA class switching in the human intestine. Follicular mantle (FM) naive $\operatorname{lgM}^{+} \lg D^{+} B$ cells undergo TD direct IgM-to-lgA1 CSR as well as SHM in the GC of PPs upon exposure to CD4 ${ }^{+}$T-helper cells expressing CD40L and cytokines, including (data not shown) IL-10 and TGF- $\beta$. The resulting mutated IgA $1^{+} B$ cells migrate into the colon LP, where they undergo TI sequential IgA1-to-lgA2 CSR under the influence of APRIL and IL-10 released by intestinal epithelial cells (IECs). IECs produce these innate B-cell-stimulating cytokines after sensing bacterial molecular patterns (BMPs) through TLRs. IECs further amplify APRIL and IL-10 production by stimulating LP dendritic cells (DCs) through an IL-7-like cytokine known as thymic stromal lymphopoietin (TSLP) (discussed in ref. 2). Then, protease-resistant IgA2 dimers released by LP B cells undergo plgR-mediated transcytosis to prevent adhesion of commensal bacteria to IECs. Additional IgA2 would originate from PP- or bone marrow-derived $\operatorname{lgM}{ }^{+} \lg D^{+} B$ cells, which undergo TI IgM-to-lgA2 CSR in the APRIL-rich environment of the LP. These $B$ cells likely include both mutated and unmutated subsets. APRIL, a proliferation-inducing ligand; IgA, immunoglobulin A; CSR, class switch DNA recombination; GC, germinal center; LP, lamina propria; plgR, polymeric Ig receptor; PPs, Peyer's patches; SHM, somatic hypermutation; TD, T-cell-dependent; TI, T-cell-independent ;TLRs, Toll-like receptors.

high-affinity IgA. ${ }^{10}$ High-affinity IgA also originates from mesenteric lymph nodes and isolated lymphoid follicles (reviewed in ref. 1), underscoring the variegate nature of mucosal environments supporting TD IgA responses.

Although important, follicular structures are not absolutely required for mucosal IgA responses (reviewed in ref. 1). Indeed, mice lacking CD28, a T-cell molecule necessary for GC formation, exhibit conserved intestinal but impaired systemic TD IgA responses. Similarly, mice lacking the Id 2 transcription factor or the retinoic acid-related orphan receptor $\gamma$, as well as bone marrow-reconstituted mice lacking lymphotoxin- $\alpha$ or both lymphotoxin- $\alpha$ and tumor necrosis factor, do not develop mucosal follicles and yet retain intestinal IgA production. Altogether, these data imply that the gutassociated lymphoid tissue initiates TD IgA responses through both follicular and extrafollicular pathways.

Extrafollicular pathways are essential for mediating quick TI production of low- affinity IgA antibodies against commensal bacteria and dietary antigens. In mice, this response targets highly conserved antigens through poorly diversified antibodies predominantly produced by B-1 cells (reviewed in ref. 1). This B-cell subset, which is lodged in the peritoneal cavity, does not require help from $\mathrm{CD}_{4}{ }^{+}$ $T$ cells via $\mathrm{CD} 40 \mathrm{~L}$, and releases polyreactive IgA in addition to IgM. MacPherson and $\mathrm{Uhr}^{11}$ found that B-1 cells undergo TI IgA production upon interacting with dendritic cells loaded with commensal bacteria. In agreement with these data, data from Rescigno's group ${ }^{12}$ show that intestinal dendritic cells sample lumenal antigens through transepithelial projections. Such sampling activity likely results in Toll-like receptor-dependent upregulation of innate IgA CSR-inducing factors, including APRIL and its homologue B-cell-activating factor of the tumor necrosis factor family (BAFF, also known as BLyS), ${ }^{2,13}$

Although lacking canonical B-1 cells, human B cells undergo CD40-independent IgA CSR upon exposure to BAFF and APRIL. ${ }^{14}$ These TI stimuli may be more prominent than originally anticipated, as up to $10 \%$ of patients with common variable immunodeficiency or selective IgA deficiency exhibit defective signaling via transmembrane activator and calcium modulator and cyclophylin ligand interactor, a BAFF and APRIL receptor expressed by B cells. ${ }^{15,16}$ Conversely, patients with hyper-IgM syndrome caused by CD40 signaling defects show conserved systemic and/or mucosal IgA responses. ${ }^{2,17}$ Yet, these patients lack functional GCs, suggesting that humans, like mice, have developed extrafollicular pathways for TI IgA production. The human B-cell types involved in TI IgA responses remain to be identified, but may include IgM memory B cells, which make mutated antibodies against bacterial polysaccharides, as well as transitional B cells, which make unmutated antibodies against multiple antigenic specificities. ${ }^{18,19}$ Ongoing studies in our lab are exploring the gut-homing properties of these B-cell subsets.

The following observations indicate that the intestinal LP can serve as an IgAinductive site in both mice and humans. First, the LP contains IgM ${ }^{+} \mathrm{B}$ cells that 
can further switch to IgA under the influence of powerful B-cell-stimulating factors released by epithelial cells, dendritic cells, and stromal cells, including BAFF and APRIL. ${ }^{2,20}$ Most of these IgM ${ }^{+} B$ cells likely derive from PPs, isolated lymphoid follicles, and mesenteric lymph nodes, whereas others may originate from the peritoneal cavity and the bone marrow (reviewed in ref. 1). Second, the LP contains $\operatorname{IgA}{ }^{+} \mathrm{B}$ cells bearing hallmarks of active IgA CSR, including AID and postdeletional switch circle transcripts, as reported independently by three groups in different experimental systems. ${ }^{2-4}$ Third, the presence of IgA CSR and AID in the LP is in agreement with growing evidence showing that AIDdependent diversification of Ig genes is not restricted to the GC, but also occurs in extrafollicular areas, including the mucosal subepithelium. ${ }^{13,21}$

Unlike other groups, ${ }^{2-4}$ Kiyono, Spencer, and Lycke's groups ${ }^{5-7}$ could not detect AID and IgA switch circle transcripts in mouse or human LP B cells. Discrepancies might be due to the demanding nature of the experiments required for the identification of AID and switch circle transcripts. Indeed, these experiments need to circumvent poor cellular yields and low amounts of specific CSRassociated transcripts. The fact that the mouse gut contains a less abundant and diverse microflora than the human gut may account for the differences between species. Additional differences may originate from the fact that the small intestine, which is often studied in mice, is less exposed to bacteria and may therefore contain less IgA class-switching LP B cells than the large intestine. Another important consideration relates to the possible existence of multiple mucosal microcompartments for TI IgA production, including the perifollicular areas of PPs and mesenteric lymph nodes. These sites may become predominant under specific experimental conditions. Finally, it must be noted that, Spencer and Lycke's groups ${ }^{6,7}$ although unable to detect AID and switch circle transcripts, detected germline $\mathrm{C} \alpha$ transcripts in LP B cells. These transcripts are much more abundant than switch circle transcripts and are usually associated with ongoing IgA CSR.
By ensuring the establishment of complex commensal and symbiotic relationships, co-evolution of mammals and bacteria over the past 200 million years has contributed to the development of multiple follicular and extrafollicular layers of protection in the intestinal mucosa. By documenting IgA class switching in the LP, recent studies have identified an additional extrafollicular layer and blurred the distinction between sites of adaptive response induction and deployment of effector activities. ${ }^{2-4}$ While PPs, isolated lymphoid follicles, and mesenteric lymph nodes must be certainly regarded as major inductive sites for TD production of high-affinity IgA against pathogens, the LP as well as other as yet undefined compartments could account for TI production of low-affinity IgA against commensals and dietary antigens. Further studies are warranted to understand better the composition and regulation of each mucosal site and its relative contribution to the induction of mono- and polyspecific IgA responses.

\section{DISCLOSURE}

The author declared no conflict of interest.

\section{2008 Society for Mucosal Immunology}

\section{REFERENCES}

1. Suzuki, K., Ha, S.A., Tsuii, M. \& Fagarasan, S. Intestinal IgA synthesis: a primitive form of adaptive immunity that regulates microbial communities in the gut. Semin. Immunol. 19, 127-135 (2007).

2. He, B., Xu, W., Santini, P.A., Polydorides, A.D., Chiu, A., Estrella, J. et al. Intestinal bacteria trigger $T$ cell-independent immunoglobulin A(2) class switching by inducing epithelial-cell secretion of the cytokine APRIL. Immunity $\mathbf{2 6}$, 812-826 (2007).

3. Crouch, E.E., Li, Z., Takizawa, M., Fichtner-Feigl, S., Gourzi, P., Montano, C. et al. Regulation of AID expression in the immune response. J. Exp. Med. 204, 1145-1156 (2007).

4. Fagarasan, S., Kinoshita, K., Muramatsu, M., Ikuta, K. \& Honjo, T. In situ class switching and differentiation to IgA-producing cells in the gut lamina propria. Nature 413, 639-643 (2001).

5. Shikina, T., Hiroi, P., Iwatani, K., Jang, M.H., Fukuyama, S., Tamura, M. et al. IgA class switch occurs in the organized nasopharynx- and gutassociated lymphoid tissue, but not in the diffuse lamina propria of airways and gut. J. Immunol. 172, 6259-6264 (2004)

6. Boursier, L., Gordon, J.N., Thiagamoorthy, S., Edgeworth, J.D. \& Spencer, J. Human intestinal IgA response is generated in the organized gutassociated lymphoid tissue but not in the lamina propria. Gastroenterology 128, 1879-1889 (2005).

7. Bergqvist, P., Gardby, E., Stensson, A., Bemark, M. \& Lycke, N.Y. Gut IgA class switch recombination in the absence of CD40 does not occur in the lamina propria and is independent of germinal centers. J. Immunol. 177, 7772-7783 (2006).

8. Honjo, T., Kinoshita, K. \& Muramatsu, M. Molecular mechanism of class switch recombination: linkage with somatic hypermutation. Annu. Rev. Immunol. 20, 165-196 (2002).

9. Cazac, B.B. \& Roes, J. TGF-beta receptor controls $B$ cell responsiveness and induction of IgA in vivo. Immunity 13, 443-451 (2000).

10. Mora, J.R., Iwata, M., Eksteen, B., Song, S.Y., Junt, T., Senman, B. et al. Generation of guthoming IgA-secreting B cells by intestinal dendritic cells. Science 314, 1157-1160 (2006).

11. Macpherson, A.J. \& Uhr, T. Induction of protective lgA by intestinal dendritic cells carrying commensal bacteria. Science $\mathbf{3 0 3}$, 1662-1665 (2004).

12. Rescigno, M., Urbano, M., Valzasina, B., Francolini, M., Rotta, G., Bonasio, R. et al. Dendritic cells express tight junction proteins and penetrate gut epithelial monolayers to sample bacteria. Nat. Immunol. 2, 361-367 (2001).

13. Xu, W., He, B., Chiu, A., Chandburn, A., Shan, M., Buldys, M. et al. Epithelial cells trigger frontline immunoglobulin class switching through a pathway regulated by the inhibitor SLPI. Nat. Immunol. 8, 294-303 (2007).

14. Litinskiy, M.B., Nardelli, B., Hilbert, D.M., He, B., Schaffer, A., Casali, P. et al. DCs induce CD40-independent immunoglobulin class switching through BLyS and APRIL. Nat. Immunol. 3, 822-829 (2002).

15. Castigli, E., Wilson, S.A., Garibyan, L., Rachid, R., Bonilla, F., Schneider, L., Geha, R.S. et al. TACl is mutant in common variable immunodeficiency and lgA deficiency. Nat. Genet. 37, 829-834 (2005).

16. Salzer, U., Chapel, H.M., Webster, A.D., Pan-Hammarstrom, Q., Schmitt-Graeff, A., Schlesier, M. et al. Mutations in TNFRSF13B encoding TACl are associated with common variable immunodeficiency in humans. Nat. Genet. 37, 820-828 (2005)

17. Jain, A., Ma, C.A., Lopez-Granados, E., Means, G., Brady, W., Orange, J.S. et al. Specific NEMO mutations impair CD40mediated c-Rel activation and B cell terminal differentiation. J. Clin. Invest. 114, 1593-1602 (2004)

18. Wardemann, H., Yurasov, S., Schaefer, A., Young, J.W., Meffre, E., Nussenzweig, M.C. et al. Predominant autoantibody production by early human B cell precursors. Science $\mathbf{3 0 1}$, 1374-1377 (2003).

19. Weller, S., Braun, M.C., Tan, B.K., Rosenwald, A., Cordier, C., Conley, M.E. et al. Human blood IgM "memory" B cells are circulating splenic marginal zone B cells harboring a prediversified immunoglobulin repertoire. Blood 104, 3647-3654 (2004).

20. Tezuka, H., Abe, Y., Iwata, M., Takeuchi, H., Ishikawa, H., Matsushita, M. et al. Regulation of IgA production by naturally occurring TNF/ iNOS-producing dendritic cells. Nature 448, 929-933 (2007).

21. MacLennan, Toellner, K.M., Cunningham, A.F., Serre, K., Sze, D.M., Zuniga, E. et al. Extrafollicular antibody responses. Immunol. Rev. 194, 8-18 (2003). 